\title{
Novel Delay-dependent Robust Stability Criteria of Delay Hopfield Neural Networks with Time-varying
}

\author{
Fang $\mathrm{Liu}^{\mathrm{a}, *}$, Min $\mathrm{Wu}^{\mathrm{b}}$, Yong $\mathrm{Li}^{\mathrm{c}}$, Yong $\mathrm{He}^{\mathrm{b}}$, Ryuichi Yokoyama ${ }^{\mathrm{d}}$ \\ ${ }^{\text {a }}$ School of Information Science and Engineering, Central South University, Changsha 410083, China. \\ ${ }^{\mathrm{b}}$ School of Automation, China University of Geosciences, Wuhan 430074, China \\ ${ }^{c}$ College of Electrical and Information Engineering, Hunan University, Changsha 410082, China. \\ ${ }^{\mathrm{d}}$ Graduate School of Environmental and Energy Engineering, Waseda University, Tokyo 162-0041, Japan. \\ *Corresponding Author: csuliufang@csu.edu.cn
}

\begin{abstract}
This paper investigates the robust stability problem of Hopfield neural networks(HNNs) with time-varying delay. Two novel LMI-based delay-dependent robust stability criteria are obtained by constructing appropriate Lyapunov-Krasovskii functional. This new criteria based on the free-weighting matrices approach prove to be less conservativeness, which not only retain any useful terms in the derivative of Lyapunov-Krasovskii functional, but also consider the relationship among the time-delay, its upper bound and their difference. Finally, some numerical examples are given to demonstrate its merits and effectiveness of the proposed methods.
\end{abstract}

Keywords: Hopfield neural networks(HNNs), delay-dependent robust stability, time-varying delay, Lyapunov-Krasovskii functional, linear matrix inequality (LMI).

\section{Introduction}

During the past two decades, the Hopfield neural networks(HNNs) system has been attracted an increasing attention since it has found extensive applications in solving some optimization problems, system controlling, signal processing and static image treatment and other areas $^{(1-3)}$. It is well known that applications of neural networks heavily depend on their dynamic behavior, but time-delays often occur in the interaction between neurons related to their dynamic behaviors. Furthermore, the existence of time-delays is usually a source of instability and deteriorated performance. Therefore, the stability analysis of has not only important theoretical interesting, but also practical value.
Up to now, via different methods, lots of sufficient conditions have been obtained to guarantee the stability of delayed HNNs stability ${ }^{(4-19)}$. Among these works, stability criteria for HNNs can be classified into two types: delay-dependent criteria and delay-independent criteria. Since delay-dependent criteria make use of information on the length of delays, they are less conservative than delay-independent ones, especially when the time delay is small. Although delay-dependent stability results for the delayed HNNs has been obtained ${ }^{(16-19)}$, they have some conservativeness to extent, for example, the time delay is time-invariant. In practice, due to the finite switching speed of amplifiers or finite speed of information processing , time delay is often time-varying, which remains a space for further research.

Recently, a free-weighting matrix approach ${ }^{(20,21)}$ has been successfully employed to study the stability problem for neural networks and lots of less conservative conditions are obtained. Enlightened by it, in this paper, two improved LMI-based delay-dependent robust stability criteria are obtained for HNNs system with time-varying delay and constant delay by constructing appropriate Lyapunov-Krasovskii functional, which retain any useful terms in the derivative of Lyapunov-Krasovskii functional and consider the relationship among the time-delay, its upper bound and their difference. Finally, some numerical examples are given to demonstrate its advantages and effectiveness of the proposed methods.

Notation: Throughout this paper, the superscripts ' $T$ ' and ' -1 ' stand for the transpose and the inverse of a matrix; respectively; $\mathfrak{R}^{n}$ denotes the $n$-dimensional Euclidean space; $P>0$ means that the matrix $P$ is positive definite; $I$ is an appropriately dimensioned identity matrix; $\operatorname{diag}\{\cdots\}$ denotes a block-diagonal matrix; $\|z\|$ is the Euclidean norm of $z$; and the symmetric matrix are denoted by *, e.g., 
$\left[\begin{array}{ll}X & Y \\ * & Z\end{array}\right]=\left[\begin{array}{cc}X & Y \\ Y^{T} & Z\end{array}\right]$

\section{Problem formulation}

Consider the following delayed Hopfield neural networks(HNNs):

$$
\dot{x}(t)=-C x(t)+A g(x(t-d(t)))+u
$$

where $x(\cdot)=\left[x_{1}(\cdot), x_{2}(\cdot), \cdots, x_{n}(\cdot)\right]^{T} \in \mathfrak{R}^{n}$ is the neuron state vector; $\quad g(x(\cdot))=\left[g\left(x_{1}(\cdot)\right), g\left(x_{2}(\cdot)\right), \cdots, g\left(x_{n}(\cdot)\right)\right]^{T} \in \mathfrak{R}^{n}$ denotes the neuron activation function; $u=\left[u_{1}, u_{2}, \cdots, u_{n}\right]^{T} \in \mathfrak{R}^{n}$ is a constant input vector; $C=\operatorname{diag}\left\{c_{1}, c_{2}, \cdots, c_{n}\right\}$ is a diagonal matrix with $c_{i}>0$; and $A$ is the delayed connection weight matrix. $d(t)$ is the time delay. We will consider the following two cases for the time delay.

Case 1: $d(t)$ is a time-varying delay satisfying for all $t>0$ :

$$
\begin{aligned}
& 0 \leq d(t) \leq h \\
& \dot{d}(t) \leq \mu
\end{aligned}
$$

where $\mu$ and $h$ are constants.

Case 2: $d(t)$ is a time-invariant delay satisfying for all $t>0$ :

$$
0 \leq d(t)=d \leq h
$$

where $h$ is constant.

In addition, it is assumed that each neuron activation function in system $(1), g_{j}(\cdot), j=1,2, \cdots n$, satisfies the following condition:

$$
0 \leq \frac{g_{j}(x)-g_{j}(y)}{x-y} \leq L_{j}, \quad \forall x, y \in \mathfrak{R}, x \neq y, j=1,2, \cdots, n
$$

where $L_{j}, j=1,2, \cdots, n$ are positive constants.

Let a vector $x^{*}=\left[x_{1}^{*}, x_{2}^{*}, \cdots, x_{n}^{*}\right]^{T}$ be an equilibrium point of equation (1). By the transformation $z(\cdot)=x(\cdot)-x^{*}$, one can shift the equilibrium point $x^{*}$ of equation (1) to the origin, and consequently, system (1) can be rearranged as

$$
\dot{z}(t)=-C z(t)+A f(x(t-d(t)))
$$

where

$$
z(\cdot)=\left[z_{1}(\cdot), z_{2}(\cdot), \cdots, z_{n}(\cdot)\right]^{T}
$$

is the state vector of the transformed system, and $f(z(\cdot))=\left[f\left(z_{1}(\cdot)\right), f\left(z_{2}(\cdot)\right), \cdots, f\left(z_{n}(\cdot)\right)\right]^{T}$ with $f_{j}\left(z_{j}(\cdot)\right)=g_{j}\left(z_{j}(\cdot)+z_{j}^{*}\right)-g_{j}\left(z_{j}^{*}\right), j=1,2, \cdots, n$.

Note that the functions $f_{j}(\cdot), j=1,2, \cdots, n$ satisfy the following condition:

$0 \leq \frac{f_{j}\left(z_{j}\right)}{z_{j}} \leq L_{j}, \quad f_{j}(0)=0, \forall z_{j} \in \Re, j=1,2, \cdots, n$

which is equivalent to

$f_{j}\left(z_{j}\right)\left[f_{j}\left(z_{j}\right)-L_{j} z_{j}\right] \leq 0, \quad f_{j}(0)=0, j=1,2, \cdots, n$

The following lemmas will be employed to derive the new criterion.

Lemma 1: For any vectors $a, b \in \mathfrak{R}^{n}$, the inequality

$$
2 a^{T} b \leq a^{T} X a+b^{T} X^{-1} b
$$

Lemma 2: [9] Assuming that (7) holds, then $\int_{v}^{u}\left[f_{i}(s)-f_{j}(s)\right] d s \leq[u-v]\left[f_{i}(u)-f_{i}(v)\right], \quad j=1,2, \cdots, n$.

\section{Delay-dependent asymptotic stability criteria}

In the following, two new asymptotic stability criteria are derived by the free-weighting matrix approach.

Theorem 1: Under case 1, for given scalars $h \geq 0$ and $\mu$, the origin of system (6) with (7) and a time-varying delay satisfying conditions (2) and (3) is globally asymptotically stable if there exist $P=P^{T}>0$, $Q_{i}=Q_{i}^{T}>0, i=1,2,3, Z=Z^{T}>0, X=\left[\begin{array}{cc}X_{11} & X_{21} \\ * & X_{22}\end{array}\right] \geq 0$, $\Lambda=\operatorname{diag}\left(\lambda_{1}, \lambda_{2}, \cdots, \lambda_{n}\right), R=\operatorname{diag}\left(r_{1}, r_{2}, \cdots, r_{n}\right)$,

$S=\operatorname{diag}\left(s_{1}, S_{2}, \cdots, s_{n}\right)$, and any appropriately dimensioned matrices $T_{i}, i=1,2, N=\left[N_{1}^{T} N_{2}^{T}\right]^{T}$, and $M=\left[M_{1}^{T} M_{2}^{T}\right]^{T}$ such that the following LMIs are feasible:

$\Phi=\left[\begin{array}{cccccc}\Phi_{11} & \Phi_{12} & \Phi_{13} & -M_{1} & L R & -T_{1} A \\ * & \Phi_{22} & 0 & 0 & \Lambda & -T_{2} A \\ * & * & \Phi_{33} & -M_{2} & 0 & L S \\ * & * & * & -Q_{3} & 0 & 0 \\ * & * & * & * & Q_{2}-2 R & 0 \\ * & * & * & * & * & -(1-\mu) Q_{2}-2 S\end{array}\right]<0$

$$
\begin{aligned}
& \Psi_{1}=\left[\begin{array}{ll}
X & N \\
* & Z
\end{array}\right] \geq 0 \\
& \Psi_{2}=\left[\begin{array}{cc}
X & M \\
* & Z
\end{array}\right] \geq 0
\end{aligned}
$$

where 


$$
\begin{aligned}
& \Phi_{11}=Q_{1}+Q_{3}+N_{1}+N_{1}^{T}+T_{1} C+C^{T} T_{1}^{T}+h X_{11} \\
& \Phi_{12}=P+T_{1}+C^{T} T_{2}^{T} \\
& \Phi_{13}=-N_{1}+N_{2}^{T}+M_{1}+h X_{12} \\
& \Phi_{22}=h Z+T_{2}+T_{2}^{T} \\
& \Phi_{33}=-(1-\mu) Q_{1}-N_{2}-N_{2}^{T}+M_{2}+M_{2}^{T}+h X_{22} \\
& L=\operatorname{diag}\left\{l_{1}, l_{2}, \cdots l_{n}\right\} .
\end{aligned}
$$

Proof: Construct the following Lyapunov-Krasovskii functional candidate:

$$
\begin{aligned}
& V(z(t))=V_{1}(z(t))+V_{2}(z(t))+V_{3}(z(t)) \\
& V_{1}(z(t))=z^{T}(t) P z(t)+2 \sum_{i=1}^{n} \lambda_{i} \int_{0}^{z_{j}} f_{i}(s) d s \\
& V_{2}(z(t))=\int_{t-d(t)}^{t}\left[z^{T}(s) Q_{1} z(s)+f^{T}(z(s)) Q_{2} f(z(s))\right] d s \\
& V_{3}(z(t))=\int_{t-h}^{t} z^{T}(s) Q_{3} z(s) d s \\
& V_{4}(z(t))=\int_{-h}^{0} \int_{t+\theta}^{t} \dot{z}^{T}(s) Z \dot{z}(s) d s d \theta
\end{aligned}
$$

where $P=P^{T}>0, Q_{i}=Q_{i}^{T}>0, i=1,2,3, Z=Z^{T}>0$, and $\Lambda=\operatorname{diag}\left(\lambda_{1}, \lambda_{2}, \cdots, \lambda_{n}\right) \geq 0$ are to be determined.

Then calculating the derivatives of $V_{i}(z(t)), i=1,2,3$ defined in (14) along the trajectories of system (6) and using (15) yields:

$$
\begin{aligned}
& \dot{V}_{1}(z(t))=2 z^{T}(t) P \dot{z}(t)+2 f^{T}(z(t)) \Lambda \dot{z}(t) \\
& V_{2}(z(t)) \leq {\left[z^{T}(t) Q_{1} z(t)+f^{T}(z(t)) Q_{2} f(z(t))\right] } \\
&-(1-\mu)\left[z^{T}(t-d(t)) Q_{1} z(t-d(t))\right. \\
& V_{3}(z(t))= z^{T}(t) Q_{3} z(t)--z^{T}(t-h) Q_{3} z(t-h) \\
& V_{4}(z(t))= h \dot{z}^{T}(t) Z \dot{z}(t)-\int_{t-d(t)}^{t} \dot{z}^{T}(s) Z \dot{z}(s) d s \\
&+\int_{t-h}^{t-d(t)} \dot{z}^{T}(s) Z \dot{z}(s) d s
\end{aligned}
$$

For any appropriately dimensioned matrices $T_{i}, i=1,2$, the following holds:

$0=2\left[z^{T}(t) T_{1}+\dot{z}^{T}(t) T_{2}\right][\dot{z}(t)+C z(t)-A f(x(t-d(t)))]$

On the other hand, using the Leibniz-Newton formula, for any appropriately dimensioned matrices $N, M$, the following equations are true:

$$
\begin{array}{r}
0=2 \xi_{1}^{T}(t) N\left[z(t)-z(t-d(t))-\int_{t-d(t)}^{t} \dot{z}(s) d s\right] \\
0=2 \xi_{1}^{T}(t) M\left[z(t-d(t))-z(t-h)-\int_{t-h}^{t-d(t)} \dot{z}(s) d s\right]
\end{array}
$$

where

$$
\xi_{1}(t)=\left[z^{T}(t), z^{T}(t-d(t))\right]^{T} .
$$

In addition, for any semi-positive definite matrix $X=X^{T}=\left[\begin{array}{cc}X_{11} & X_{12} \\ * & X_{22}\end{array}\right] \geq 0$, the following equation holds:

$0=\int_{t-h}^{t} \xi_{1}^{T}(t) X \xi_{1}(t) d s-\int_{t-h}^{t} \xi_{1}^{T}(t) X \xi_{1}(t) d s$ $=h \xi_{1}^{T}(t) X \xi_{1}(t)-\int_{t-d(t)}^{t} \xi_{1}^{T}(t) X \xi_{1}(t) d s+\int_{t-h}^{t-d(t)} \xi_{1}^{T}(t) X \xi_{1}(t) d s$

$$
\text { For any } \quad R=\operatorname{diag}\left(r_{1}, r_{2}, \cdots, r_{n}\right) \geq 0 \quad \text { and }
$$
$S=\operatorname{diag}\left(s_{1}, s_{2}, \cdots, s_{n}\right) \geq 0$, It is clear from (8) that the following inequality are true:

$$
\begin{aligned}
0 & \leq 2 z^{T}(t) L R f(z(t))-2 f^{T}(z(t)) R f(z(t)) \\
& +2 z^{T}(t-d(t)) L S f(z(t-d(t))) \\
& -2 f^{T}(z(t-d(t))) S f(z(t-d(t))) .
\end{aligned}
$$

Thus, adding the terms on the right side of (19)-(23) to $\dot{V}(z(t))$ yields:

$$
\begin{aligned}
\dot{V}(z(t)) & \leq 2 z^{T}(t) P \dot{z}(t)+2 f^{T}(z(t)) \Lambda \dot{z}(t) \\
& +\left[z^{T}(t)\left(Q_{1}+Q_{3}\right) z(t)+f^{T}(z(t)) Q_{2} f(z(t))\right] \\
& -(1-\mu)\left[z^{T}(t-d(t)) Q_{1} z(t-d(t))\right. \\
& \left.+f^{T}(z(t-d(t))) Q_{2} f(z(t-d(t)))\right] \\
& -z^{T}(t-h) Q_{3} z(t-h)+h \dot{z}^{T}(t) Z \dot{z}(t) \\
& -\int_{t-d(t)}^{t} \dot{z}^{T}(s) Z \dot{z}(s) d s+\int_{t-h}^{t-d(t)} \dot{z}^{T}(s) Z \dot{z}(s) d s \\
& +\xi^{T}(t) \Phi \xi(t)-\int_{t-d(t)}^{t} \eta^{T}(t, s) \Psi_{1} \eta(t, s) d s \\
& -\int_{t-h}^{t-d(t)} \eta^{T}(t, s) \Psi_{2} \eta(t, s) d s \\
& +2\left[z^{T}(t) T_{1}+\dot{z}^{T}(t) T_{2}\right][\dot{z}(t)+C z(t)-A f(x(t-d(t)))] \\
& +2 \xi_{1}^{T}(t) N\left[z(t)-z(t-d(t))-\int_{t-d(t)}^{t} \dot{z}(s) d s\right] \\
& +2 \xi_{1}^{T}(t) M\left[z(t-d(t))-z(t-h)-\int_{t-h}^{t-d(t)} \dot{z}(s) d s\right] \\
& +h \xi_{1}^{T}(t) X \xi_{1}(t)-\int_{t-d(t)}^{t} \xi_{1}^{T}(t) X \xi_{1}(t) d s \\
+ & \int_{t-h}^{t-d(t)} \xi_{1}^{T}(t) X \xi_{1}(t) d s \\
+ & 2 z^{T}(t) L R f(z(t))-2 f^{T}(z(t)) R f(z(t)) \\
+ & 2 z^{T}(t-d(t)) L S f(z(t-d(t))) \\
+ & 2 f^{T}(z(t-d(t))) S f(z(t-d(t)))
\end{aligned}
$$

where 


$$
\begin{aligned}
\xi(t)=\left[z^{T}(t), \dot{z}^{T}(t), z^{T}(t-d(t)), z^{T}(t-h),\right. \\
\left.f^{T}(z(t)), f^{T}(z(t-d(t)))\right]^{T}
\end{aligned}
$$

$\eta(t, s)=\left[\xi_{1}^{T}(t), \dot{z}^{T}(s)\right]^{T}$

and $\Phi$ and $\Psi_{i}, i=1,2$ are defined in(11)-(13), if $\Phi<0$

and $\Psi_{i} \geq 0, i=1,2$, then $\dot{V}(z(t)) \leq 0$ for any $\xi(t) \neq 0$.

This completes the proof.

Remark 1: Theorem 1 provides a new delay-dependent global asymptotic stability condition for the delayed HNNs in terms of an LMI, which can deal with the case for HNNs system with time-varying delay.

Now, we provide a stability condition for the HNN system under case 2.

Theorem 2: Under case 2, for given scalars $h \geq 0$, the origin of system (6) with (7) and a time delay satisfying conditions (4) is globally asymptotically stable if there exist, $P=P^{T}>0, \quad Q_{i}=Q_{i}^{T}>0, i=1,2,3, Z=Z^{T}>0$,

$X=\left[\begin{array}{cc}X_{11} & X_{21} \\ * & X_{22}\end{array}\right] \geq 0, \Lambda=\operatorname{diag}\left(\lambda_{1}, \lambda_{2}, \cdots, \lambda_{n}\right)$,

$R=\operatorname{diag}\left(r_{1}, r_{2}, \cdots, r_{n}\right), S=\operatorname{diag}\left(s_{1}, s_{2}, \cdots, s_{n}\right)$,

and any appropriately dimensioned matrices $T_{i}, i=1,2, N=\left[N_{1}^{T} N_{2}^{T}\right]^{T}$, and $M=\left[M_{1}^{T} M_{2}^{T}\right]^{T}$ such that the following LMIs are feasible:

$\Phi=\left[\begin{array}{cccccc}\Phi_{11} & \Phi_{12} & \Phi_{13} & -M_{1} & L R & -T_{1} A \\ * & \Phi_{22} & 0 & 0 & \Lambda & -T_{2} A \\ * & * & \Phi_{33} & -M_{2} & 0 & L S \\ * & * & * & -Q_{3} & 0 & 0 \\ * & * & * & * & Q_{2}-2 R & 0 \\ * & * & * & * & * & Q_{2}-2 S\end{array}\right]<0$

$$
\begin{aligned}
& \Psi_{1}=\left[\begin{array}{ll}
X & N \\
* & Z
\end{array}\right] \geq 0 \\
& \Psi_{2}=\left[\begin{array}{cc}
X & M \\
* & Z
\end{array}\right] \geq 0
\end{aligned}
$$

where

$$
\begin{aligned}
& \Phi_{11}=Q_{1}+Q_{3}+N_{1}+N_{1}^{T}+T_{1} C+C^{T} T_{1}^{T}+h X_{11} \\
& \Phi_{12}=P+T_{1}+C^{T} T_{2}^{T} \\
& \Phi_{13}=-N_{1}+N_{2}^{T}+M_{1}+h X_{12} \\
& \Phi_{22}=h Z+T_{2}+T_{2}^{T} \\
& \Phi_{33}=Q_{1}-N_{2}-N_{2}^{T}+M_{2}+M_{2}^{T}+h X_{22} \\
& L=\operatorname{diag}\left\{l_{1}, l_{2}, \cdots l_{n}\right\} .
\end{aligned}
$$

Proof: Construct the following Lyapunov-Krasovskii functional candidate:

$$
\begin{aligned}
& V(z(t))=V_{1}(z(t))+V_{2}(z(t))+V_{3}(z(t))+V_{4}(z(t)) \\
& V_{1}(z(t))=z^{T}(t) P z(t)+2 \sum_{i=1}^{n} \lambda_{i} \int_{0}^{z_{j}} f_{i}(s) d s \\
& V_{2}(z(t))=\int_{t-d}^{t}\left[z^{T}(s) Q_{1} z(s)+f^{T}(z(s)) Q_{2} f(z(s))\right] d s \\
& V_{3}(z(t))=\int_{t-h}^{t} z^{T}(s) Q_{3} z(s) d s \\
& V_{4}(z(t))=\int_{-h}^{0} \int_{t+\theta}^{t} \dot{z}^{T}(s) Z \dot{z}(s) d s d \theta
\end{aligned}
$$

where $P=P^{T}>0, Q_{i}=Q_{i}^{T}>0, i=1,2,3, Z=Z^{T}>0$, and $\Lambda=\operatorname{diag}\left(\lambda_{1}, \lambda_{2}, \cdots, \lambda_{n}\right) \geq 0$ are to be determined. Then following the similar line in Theorem 1 , we can obtain Theorem 2.

Remark 2: Theorem 2 provides a new delay-dependent global asymptotic stability condition for the HNNs with constant delay in terms of an LMI. This is derived by constructing an appropriate Lyapunov-Krasovskii functional different from those papers ${ }^{(18,19)}$, and in the derivative process of Lyapunov-Krasovskii functional, it retains any useful terms and considers the relationship among the time-delay, its upper bound and their difference.

\section{Numerical examples}

In this section, two numerical examples are given to demonstrate the effectiveness of the proposed method.

Example 1: Consider the following delayed HNNs system(1)

$\dot{x}_{1}(t)=-0.8 x_{1}(t)+0.1 g\left(x_{1}(t-d(t))\right)+0.3 g\left(x_{2}(t-d(t))\right)+5$
$\dot{x}_{2}(t)=-0.8 x_{2}(t)+0.9 g\left(x_{1}(t-d(t))\right)+0.1 g\left(x_{2}(t-d(t))\right)+3$

where the activation function is described by

$g(x)=0.5(|x+1|-|x-1|)$.

Then

$C=\left[\begin{array}{cc}0.8 & 0 \\ 0 & 5.3\end{array}\right] \quad A=\left[\begin{array}{ll}0.1 & 0.3 \\ 0.9 & 0.1\end{array}\right] \quad L_{1}=L_{2}=1$.

When time delay is time-invariant, this example is discussed in both of papers ${ }^{(16,17)}$. Employing the LMIs ${ }^{(16,17)}$, the maxium delay $h$ that guarantees the asymptotic stability of HNN (1) are obtained to be 0.058 and 2. However, by the Theorem 2 of this paper, it is found that for any arbitrarily large $h$ (as long as the numerical compution is reliable), HNN system (1) is stable. So it can be seem this proposed method has a less conservativeness.

On the other hand, wen time delay is time-varying, both of papers ${ }^{(16,17)}$ cannot be used to determine whether HNN 
system(1) is stable or not. But using the the Theorem 1of this paper, we can also calculate the values of the maxium delay $h$ which guarantees the asymptotic stability of HNN (1). The values of $h$ for various $\mu$ are listed in the following Table 1.

TABLE 1

\begin{tabular}{|c|c|c|c|}
\hline$\mu$ & 0.8 & 0.9 & 2 \\
\hline Theorem 1 & arbitrarily value & $\mathbf{1 4 . 6 6 0}$ & $\mathbf{3 . 0 9 1}$ \\
\hline
\end{tabular}

Example 2: Consider a delay HNN system in (6) with parameters:

$$
\begin{aligned}
& C=\left[\begin{array}{ccc}
4.1989 & 0 & 0 \\
0 & 0.7160 & 0 \\
0 & 0 & 1.9985
\end{array}\right] \\
& A=\left[\begin{array}{ccc}
-0.1052 & -0.5069 & -0.1121 \\
-0.0257 & -0.2808 & 0.0212 \\
0.1205 & -0.2153 & 0.1315
\end{array}\right] \\
& L_{1}=0.4219 L_{2}=3.8993 L_{3}=1.0160
\end{aligned}
$$

For this example, the delay-dependent condition ${ }^{(12)}$ cannot be satisfied for any arbitrarily small $h$. By the method $^{(18,19)}$, the maxium delay $h$ that guarantees the asymptotic stability of HNN system(6) are obtained to be1.7484 and 1.7644, respectively, while we can obtain the delayed HNN system(6) is asymptotically stale under the condition $h \leq 2.1423$ by Theorem 2. So our result has less conservativeness.

\section{Conclusions}

In this paper, by employing the free-weighting matrices approach, two less conservative LMIs-based asymptotic stability criteria are obtained without ignoring any useful terms in the derivative of Lyapunov functional for neural networks with time delay. Finally, two numerical examples are given to demonstrate that the proposed method is an improvement over the existing ones.

\section{Acknowledgment}

This work was supported in part by the National Natural Science Foundation of China (No.61304092), in part by the Research Fund for the Doctoral Program of Higher Education of China(No.20130162120022) and in part by the Hunan Provincial Natural Science Foundation of China (No.13JJ6004).

\section{References}

(1) J. J. Hopfield, "Neurons with graded response have collective computational properties like those of two-state neurons," Proc. Nat. Acad. Sci. USA, vol. 81, pp. 3088-3092, 1984.

(2) J. Farrell and A. Michel, "A synthesis procedure for Hopfield's continuous-time associative memory," IEEE Trans. Circuits Syst., vol. 37, pp. 877-884, 1990.

(3) Z. H. Guan and G. R. Chen, On delayed impulsive Hopfield neural networks, Neural Networks vol. 12, pp. 273-280, 1999.

(4) L.Wan and J. Sun, "Mean square exponential stability of stochastic delayed Hopfield neural networks," Physics Letters A, vol. 343, pp. 306-318, 2005.

(5) J. F. Nong, "Global Exponential Stability Of Delayed Hopfield Neural Networks", 2012 International Conference on Computer Science and Information Processing (CSIP), 193-196, 2012.

(6) B. Xu, X. Liu, and X. Liao, "Global exponential stability of high order Hopfield type neural networks," Appl. Mathemat. Computat., vol. 174, pp. 98-116, 2006.

(7) D.Yang, X. Liao, Y. Chen, S. Guo and H. Wang, "New delay-dependent global asymptotic stability criteria of delayed Hopfield neural networks" Nonlinear Analysis: Real World Applications, vol. 9, pp. 1894-1904, 2008.

(8) Z. Wang, H. Shu, J. Fang, "Robust stability for stochastic Hopfield neural networks with time delays", Nonlinear Analysis: Real World Applications, vol. 7, pp. 1119-1128, 2006.

(9) J. Zhang, P. Shi, and J. Qiu, "Novel robust stability criteria for uncertain stochastic Hopfield neural networks with time-varying delays," Nonlinear Analysis: Real World Applications, vol. 8, pp. 1349-1357, 2007.

(10) X. L. Li, and F. Y. Sun, "Stabilizing of Stochastic Interval Hopfield Neural Networks with Time-Varying Delays”, 2013 6th International Conference on Biomedical Engineering and Informatics (BMEI 2013), 773-777, 2013.

(11) J. F. Han, J. Q. Qiu, H. R. Jia, and X. T. Meng, "Robust stability for stochastic interval Hopfield neural networks with time delays”, 2010 Sixth International Conference on Natural Computation (ICNC 2010), 803-805, 2010.

(12) Q. Zhang., X. Wei and J. Xu, "Global asymptotic 
stabiltiy of Hopfield neural network with transmission delays,” Physics Letters A, vol. 318, pp.399-405, 2003.

(13) X. Z. Liu and R. Dickson, "Stability analysis of Hopfield neural networks with uncertainty," Mathematical and Computer Modelling, vol. 34, pp. 353-363, 2001.

(14) X. Y. Lou, B. Cui, "New LMI conditions for delay-dependent asymptotic stability of delayed Hopfield neural networks," Neurocomputing, vol. 69,pp. 2374-2378, 2006.

(15) H. T. Lu, "On stability of nonlinear continuous-time neural networks with delays," Neural Networks, vol. 13, pp. 1135-114, 2000.

(16) Q. Zhang., X. Wei and J. Xu, "Delay-dependent global stabiltiy condition for delayed Hopfield neural network," Nonlinear Analysis: Real world Application, vol. 8, pp.997-1002, 2007.

(17)D. G. Yang, Q. Liu, and Y. Wang, “ A novel delay-dependent global stability criterion of delayled Hopfield neural networks,” 2007 IEEE International Conference on Granular Computing, pp. 538-541, 2007.

(18) S. Y. Xu, J. Lam, D. W.C.Ho, and Y. Zhou, "Novel global asymptotic stability criteria for delayed cellular neural networks,” IEEE Trans. Circuits and Systems II, vol. 52, pp. 349-353, 2005.

(19) S. Y. Xu, J. Lam, and D. W.C.Ho, "A new LMI condition for delay-dependent asymptotic stability of delayed Hopfield neural networks," IEEE Trans. Circuits and Systems II, vol. 53, pp. 230-234, 2006.

(20) Y. He, Q. G. Wang, and M. Wu, "LMI-based stability criteria for neural networks with multiple time-varying delays,” Physical D, vol. 212, pp. 126-136, 2005.

(21) Y. He, G. P. Liu, and D. Rees, "New delay-dependent stability criteria for neutral networks with time-varying delay,” IEEE Trans. Neural. Networks., vol. 18, 310-314, 2007. 